\title{
Hybrid Reasoning Model for Strengthening the problem solving capability of Expert Systems
}

\author{
Kapil Khandelwal \\ PHD Scholar, Computer Science Department \\ Suresh Gyan Vihar University \\ Jaipur, India
}

\author{
Durga Prasad Sharma \\ Professor, AMUIT \\ MOEFDRE under UN Development Program, Ethiopia \\ Jaipur, India
}

\begin{abstract}
In this paper, we briefly outlined popular casebased reasoning combinations. More specifically, we focus on combinations of case-based reasoning with rule based reasoning, and model based reasoning. Further we examined the strengths and weaknesses of various reasoning models, case-based reasoning, rule-based reasoning and model-based reasoning, and discuss how they can be combined to form a more robust and better-performing hybrid. In a decision support system to address the variety of tasks a user performs, a single type of knowledge and reasoning method is often not sufficient. It is often necessary to determine which reasoning method would be the most appropriate for each task, and a combination of different methods has often shown the best results. In this study CBR was mixed with other RBR and MBR approaches to promote synergies and benefits beyond those achievable using CBR or other individual reasoning approaches alone. Each approach has advantages and disadvantages, which are proved to be complementary in a large degree. So, it is well-justified to combine these to produce effective hybrid approaches, surpassing the disadvantages of each component method. "KNAPS-CR" model integrates problem solving with learning from experience within an extensive model of different knowledge types. "KNAPS-CR" has a reasoning strategy which first attempts casebased reasoning, then rule-based reasoning, and, finally, modelbased reasoning. It learns from each problem solving session by updating its collection of cases, irrespective of which reasoning method that succeeded in solving the problem.
\end{abstract}

Keywords—knowledge based systems; KBS, sustained learning; problem solving; hybrid reasoning models; case based reasoning; CBR; model based reasoning; MBR; rule based reasoning; $R B R$

\section{INTRODUCTION}

Hybrid systems are universally better than conventional approaches. The combination of (two or more) different problem solving and knowledge representation methods is a very active research area in Artificial Intelligence. Hybrid Intelligent System is a combination of two techniques with more strength and less weakness. Almost every conceivable problem has been approached using some form of hybrid system. The aim is to create combined formalisms that benefit from each of their components. The effectiveness of various hybrid or integrated approaches has been demonstrated in a number of application areas. It is generally believed that complex problems are easier to solve with hybrid or integrated approaches.

Model-based reasoning (MBR) is an approach in which general knowledge is represented by formalizing the mathematical or physical relationships present in a problem domain. The CBR-MBR integration improves solution accuracy over that which is possible using either single approach.

TABLE I. HYBRID REASONING MODELS

\begin{tabular}{|c|c|c|}
\hline Domain & Tools & Reasoning Models \\
\hline Agriculture & HIDES & CBR,RBR \\
\hline Aircraft design & AIDA & CBR,RBR \\
\hline Aircraft Fleet Maintenance & IDS & CBR,RBR \\
\hline Architecture & FABEL & CBR,MBR, RBR \\
\hline Bioprocess recipes & SOPHIST & CBR,MBR \\
\hline Construction & ScheduleCoach & CBR,RBR \\
\hline $\begin{array}{l}\text { Equipment Failure } \\
\text { Analysis }\end{array}$ & EFAES & CBR,RBR \\
\hline Entomology & CARMA & CBR,MBR \\
\hline \multirow[t]{2}{*}{ Finance } & ECLAS & \multirow[t]{2}{*}{ CBR,RBR } \\
\hline & MARS & \\
\hline \multirow[t]{5}{*}{ Law } & IKBALS & \multirow[t]{5}{*}{ CBR,RBR } \\
\hline & SHYSTER-MYCIN & \\
\hline & CABARE & \\
\hline & GREBE & \\
\hline & DANIEL & \\
\hline Life Insurance & CCAR & CBR,RBR \\
\hline \multirow[t]{6}{*}{ Medicine/Medical } & $\mathrm{ICU}$ & \multirow[t]{4}{*}{ CBR,RBR } \\
\hline & AUGUSTE Project & \\
\hline & WHAT & \\
\hline & CARE-PARTNER & \\
\hline & CASEY & CBR,MBR \\
\hline & T-IDDM & CBR,MBR, RBR \\
\hline Menu planning & CAMPER & CBR,RBR \\
\hline \multirow[t]{2}{*}{ Music } & GYMEL & \multirow[t]{2}{*}{ CBR,RBR } \\
\hline & SAXEX & \\
\hline Plastic colorants & FORMTOOL & CBR,MBR \\
\hline $\begin{array}{l}\text { Personnel Performance } \\
\text { Evaluation }\end{array}$ & MCRS & CBR,RBR \\
\hline $\begin{array}{l}\text { Real-Time Marine } \\
\text { Environment Monitoring }\end{array}$ & CORMS AI & CBR,RBR \\
\hline Speech & ANAPRON & CBR,RBR \\
\hline Ultrasonic Rail Inspection & URS-CBR & CBR,RBR \\
\hline
\end{tabular}

Rules usually represent general knowledge, whereas cases encompass knowledge accumulated from specific (specialized) situations. Rule-based and case-based reasoning are two popular approaches used in intelligent systems. Each approach 
has advantages and disadvantages, which are proved to be complementary in a large degree. So, it is well-justified to combine rules and cases to produce effective hybrid approaches, surpassing the disadvantages of each component method [3] [9][10][11][12][13][15][17][18].

\section{Advantages \& Disadvantages of CBR, RBR, MBR \& HYBRID REASONING}

\section{A. Rule-based Reasoning}

The advantages of a rule-based approach include:

1) The ability to use, in a very direct fashion, experiential knowledge acquired from human experts. This is particularly important in domains that rely heavily on heuristics to manage complexity and/or missing information.

2) Rules map into state space search. Explanation facilities support debugging.

3) The separation of knowledge from control simplifies development of expert systems by enabling an iterative development process where the knowledge engineer acquires, implements, and tests individual rules.

4) Good performance is possible in limited domains. Because of the large amounts of knowledge required for intelligent problem solving, expert systems are limited to narrow domains. However, there are many domains where design of an appropriate system has proven extremely useful.

5) Good explanation facilities. Although the basic rulebased framework supports flexible, problem-specific explanations, it must be mentioned that the ultimate quality of these explanations depends upon the structure and content of the rules.

Explanation facilities differ widely between data- and goaldriven systems.

Disadvantages of rule-based reasoning include:

1) Often the rules obtained from human experts are highly heuristic in nature, and do not capture functional or modelbased knowledge of the domain.

2) Heuristic rules tend to be "brittle" and can have difficulty handling missing information or unexpected data values.

3) Another aspect of the brittleness of rules is a tendency to degrade rapidly near the "edges" of the domain knowledge. Unlike humans, rule-based systems are usually unable to fall back on first principles of reasoning when confronted with novel problems.

4) Explanations function at the descriptive level only, omitting theoretical explanations. This follows from the fact that heuristic rules gain much of their power by directly associating problem symptoms with solutions, without requiring (or supporting) deeper reasoning.

5) The knowledge tends to be very task dependent. Formalized domain knowledge tends to be very specific in its applicability. Currently, knowledge representation languages do not approach the flexibility of human reasoning [4] [9] [11].

\section{B. Case-based Reasoning}

The advantages of case-based reasoning include:

1) The ability to encode historical knowledge directly. In many domains, cases can be obtained from existing case histories, repair logs, or other sources, eliminating the need for intensive knowledge acquisition with a human expert.

2) Allows shortcuts in reasoning. If an appropriate case can be found, new problems can often be solved in much less time than it would take to generate a solution from rules or models and search.

3) It allows a system to avoid past errors and exploit past successes. CBR provides a model of learning that is both theoretically interesting and practical enough to apply to complex problems.

4) Extensive analysis of domain knowledge is not required. Unlike a rule-based system, where the knowledge engineer must anticipate rule interactions, CBR allows a simple additive model for knowledge acquisition. This requires an appropriate representation for cases, a useful retrieval index, and a case adaptation strategy.

5) Appropriate indexing strategies add insight and problem-solving power. The ability to distinguish differences in target problems and select an appropriate case is an important source of a case-based reasoner's power; often, indexing algorithms can provide this functionality automatically.

The disadvantages of case-based reasoning include:

1) Cases do not often include deeper knowledge of the domain. This handicaps explanation facilities, and in many situations it allows the possibility that cases may be misapplied, leading to poor quality or wrong advice.

2) A large case base can suffer problems from store/compute trade-offs.

3) It is difficult to determine good criteria for indexing and matching cases. Currently, retrieval vocabularies and similarity matching algorithms must be carefully hand crafted; this can offset many of the advantages CBR offers for knowledge acquisition [1][2][4][7][8][14].

\section{Model-based Reasoning}

The advantages of model-based reasoning include:

1) The ability to use functional/structural knowledge of the domain in problem solving. This increases the reasoner's ability to handle a variety of problems, including those that may not have been anticipated by the system's designers.

2) Model-based reasoners tend to be very robust. For the same reasons that humans often retreat to first principles when confronted with a novel problem, model based reasoners tend to be thorough and flexible problem solvers.

3) Some knowledge is transferable between tasks. Modelbased reasoners are often built using scientific, theoretical knowledge. Because science strives for generally applicable theories, this generality often extends to model-based reasoners. 
4) Often, model-based reasoners can provide causal explanations. These can convey a deeper understanding of the fault to human users, and can also play an important tutorial role.

The disadvantages of model-based reasoning include:

1) A lack of experiential (descriptive) knowledge of the domain. The heuristic methods used by rule-based approaches reflect a valuable class of expertise.

2) It requires an explicit domain model. Many domains, such as the diagnosis of failures in electronic circuits, have a strong scientific basis that supports model based approaches. However, many domains, such as some medical specialties, most design problems, or many financial applications, lack a well-defined scientific theory. Model-based approaches cannot be used in such cases.

3) High complexity. Model-based reasoning generally operates at a level of detail that leads to significant complexity; this is, after all, one of the main reasons human experts have developed heuristics in the first place.

4) Exceptional situations. Unusual circumstances, for example, bridging faults or the interaction of multiple failures in electronic components, can alter the functionality of a system in ways difficult to predict using an a priori model [4][10][12].

\section{Hybrid Design}

An important area of research and application is the combination of different reasoning models. With a hybrid architecture two or more paradigms are integrated to get a cooperative effect where the strengths of one system can compensate for the weakness of another.

In combination, we can address the disadvantages noted in the previous discussion. For example, the combination of rulebased and case-based systems can:

1) Offer a natural first check against known cases before undertaking rule-based reasoning and the associated search costs.

2) Provide a record of examples and exceptions to solutions through retention in the case base.

3) Record search-based results as cases for future use. By saving appropriate cases, a reasoner can avoid duplicating costly search.

The combination of rule-based and model-based systems can:

1) Enhance explanations with functional knowledge. This can be particularly useful in tutorial applications.

2) Improve robustness when rules fail. If there are no heuristic rules that apply to a given problem instance, the reasoner can resort to reasoning from first principles.

3) Add heuristic search to model-based search. This can help manage the complexity of model-based reasoning and allow the reasoner to choose intelligently between possible alternatives.
The combination of model-based and case-based systems can:

1) Give more mature explanations to the situations recorded in cases.

2) Offer a natural first check against stored cases before beginning the more extensive search required by model-based reasoning.

3) Provide a record of examples and exceptions in a case base that can be used to guide model-based inference.

4) Record results of model-based inference for future use [4][5][6].

\section{HyBRID REASONING MODELS}

\section{A. Sequence Models}

In this, in the first step, a rough solution is given, and in the second step, the precise solution is given by refining the rough one.

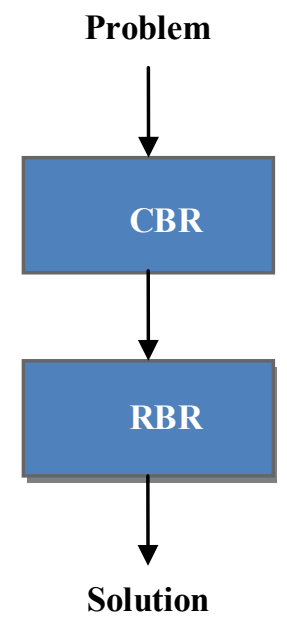

Fig. 1. CBR followed by RBR

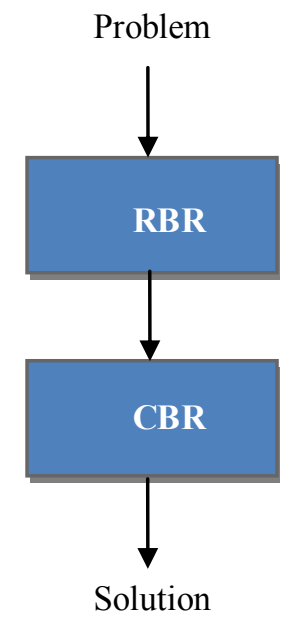

Fig. 2. RBR followed by CBR 


\section{B. Conditional Model}

In this, if the solution given in the first step is acceptable then it is used as a solution of the given problem \& otherwise next steps are invoked.

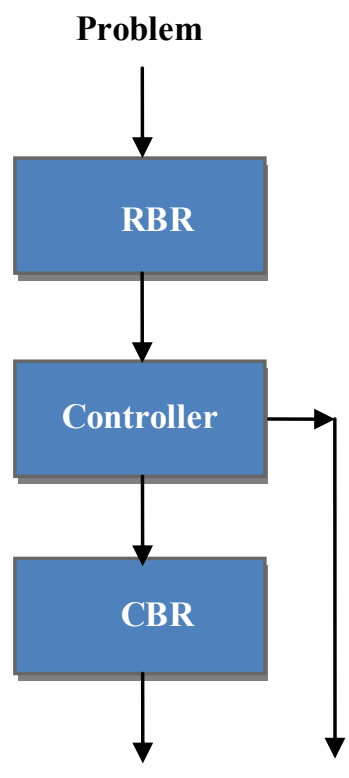

Solution

Fig. 3. RBR-Controller-CBR

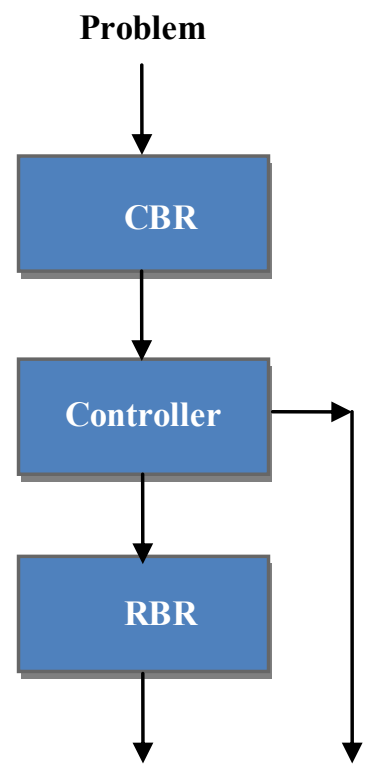

Solution

Fig. 4. CBR-Controller-RBR

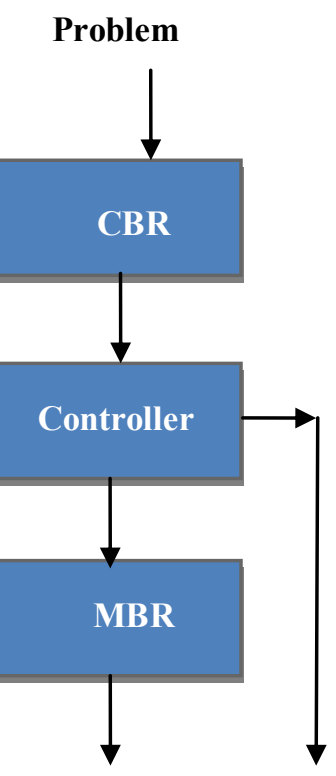

Solution

Fig. 5. CBR-Controller-MBR

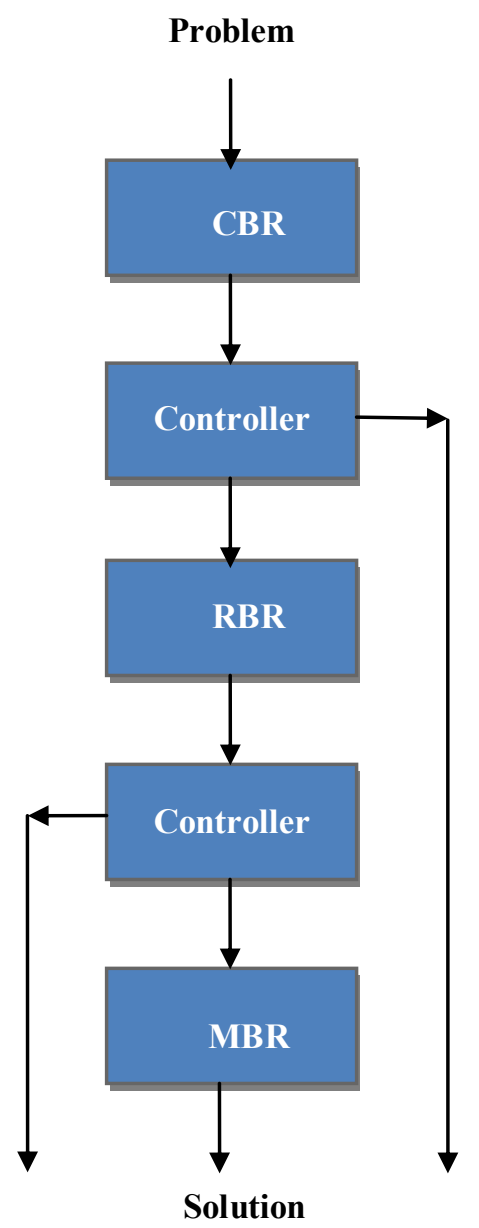

Fig. 6. CBR-Controller-RBR-Controller-MBR 


\section{FUNCTIONAL ARCHITECTURE OF "KNAPS-CR"}

"KNAPS-CR" integrates problem solving and learning into one architecture. The flow of control and information between the knowledge base and the processes of problem solving and learning in "KNAPS-CR" is shown in Figure 7.

The Figure 7 illustrates that problem solving in "KNAPSCR" is performed by a combination of model-based, casebased and rule-based reasoning (MBR, $\mathrm{CBR}$ and RBR, respectively). The learning combines case-based (CBL) and explanation-based (EBL) methods.

The process of selecting the initial reasoning paradigm starts when a set of relevant features of a problem has been identified. This feature set typically contains input features as well as inferred features, i.e. features that the system derives from the input features by using its knowledge.

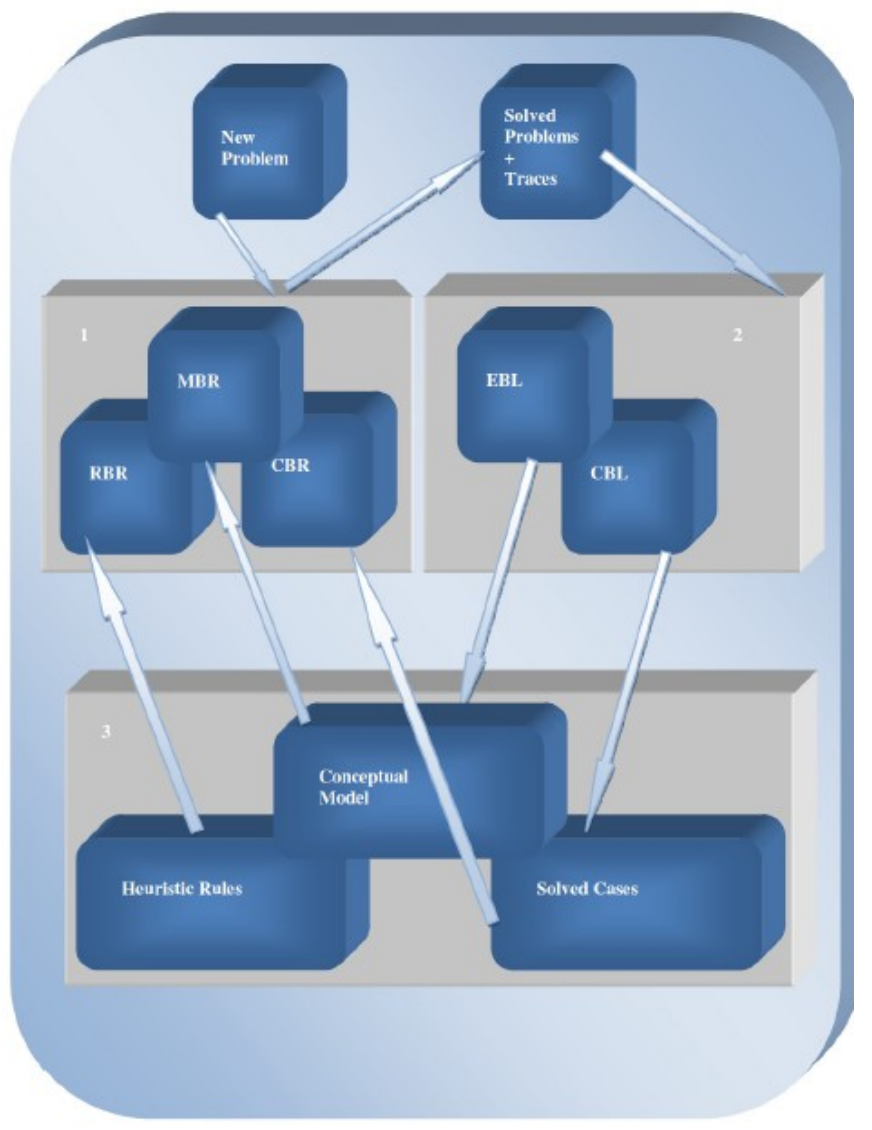

Fig. 7. KNAPS-CR's Functional Architecture - 1. Combined Reasoning; 2. Sustained Learning; 3. Knowledge Base

If the set of relevant features gives a reminding to a previous cases that is above a particular strength - called the reminding threshold - case based problem solving is tried, \& then for further refinement of the solution, the rule based reasoning is attempted. Relevant features may be input features or features inferred from the object domain model. If both the case base $\&$ the rule base fail to produce a result, the controller re-evaluates its previous decision, given the current state of the system.
If a solution was derived by modifying a previous solution, a new case is stored and difference links between the two cases are established. A new case is also created after a problem has been solved from rules or the deeper knowledge model.

Heuristic rules are integrated within the conceptual model and available for the same tasks as the conceptual domain model in general. A rule may be used to support learning.

\section{Model of COMBINED REASONING (CBR, RBR \& MBR) IN "KNAPS-CR"}

The combination of case-based $\&$ rule base method serve as the primary reasoning paradigm in "KNAPS-CR", the model based reasoning is used - as separate reasoning method - only if the combination of case-based \& rule-based methods is unable to suggest a solution.

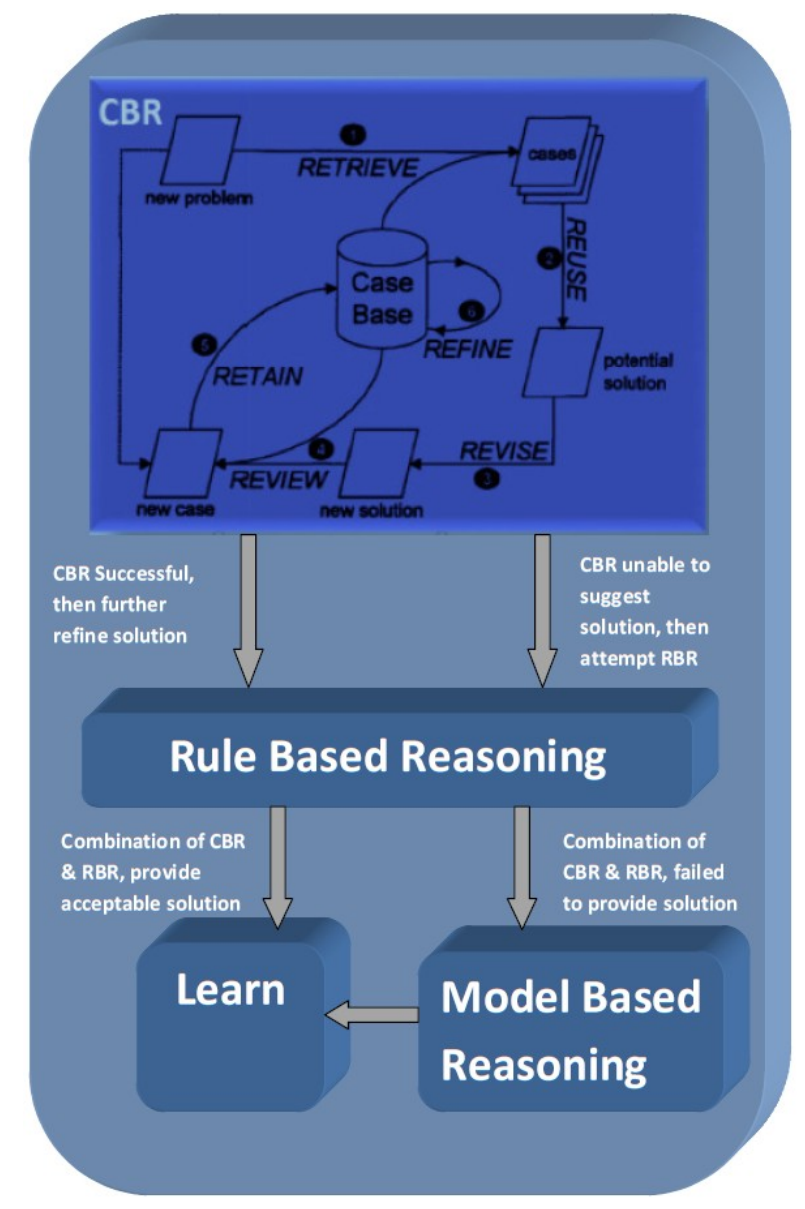

Fig. 8. Combined Reasoning in "KNAPS-CR" $(\mathrm{CBR}=$ Case-Based Reasoning, RBR = Rule-Based Reasoning, MBR = Model-Based Reasoning).

The choice of reasoning method is made after the system has gained an initial understanding of the problem. This initial understanding process (described in the next section) results in an activated problem context, including a set of relevant features for describing the problem, a structure of problem solving (sub) goals, and a hierarchy of possible faults.

The choice of reasoning method is made after the system has gained an initial understanding of the problem.This initial understanding process (described in the next section) results in 
an activated problem context, including a set of relevant features for describing the problem, a structure of problem solving (sub) goals, and a hierarchy of possible faults.

First, "KNAPS-CR" will attempt to solve the problem by case-based reasoning. The relevant findings are combined into a set of remindings, where each reminding points to a case (or a class of cases) with certain strength. If some cases are pointed to by remindings with strengths above the reminding threshold, the cases most strongly reminded of are retrieved. If no such reminding is produced, the system will trigger its rule-based reasoning method. However, before doing that it will normally try to elaborate on the findings of the cases most strongly reminded of. The purpose of this is to improve a weak match by looking for common states, constraints, etc., which will imply a stronger similarity than determined by the basic case retrieval method.

Whether the elaboration on a weak match is attempted or not depends on the strength of the strongest reminding and the size and strength of the case base relative to the rule base. If acceptable matches are found, then rule based reasoning is used to further refine the solutions obtained by case based reasoning. If no cases were reminded of in the first place, "KNAPS-CR" will also try its rule-based reasoning method, i.e. attempt to solve the problem by a combined forward chaining (from the relevant findings) and backward chaining (from the fault hierarchy) within the rule base.

The solution (fault and - possibly - treatment) is evaluated to see if it is acceptable for the current problem. If the system is unable to produce a good enough explanation to accept or reject the solution candidate, it is presented to the user for evaluation.

If for any reason the solution is unacceptable, a check is performed to determine whether the solution would be accepted if slightly modified, in which case a modification is attempted. When no more modifications are relevant and no more new cases are available for use, "KNAPS-CR" gives up case-based reasoning.

The input to a reasoning process is a problem description. This may be a description of the user's problem, or a partial solution of this problem - for example a set of descriptors which includes a fault hypothesis, given as input to the retrieval of a case containing a suitable repair.

\section{CONCLUSIONS}

In this research, we combined CBR with RBR, MBR in "KNAPS-CR" model. In our experiment and analysis, this new CBR integrated hybridized model i.e. "KNAPS-CR" model supported a wide range of tasks, including interpretation and argumentation, design and synthesis, planning, and management of long term medical conditions. Many useful synergies emerged as different reasoning strategies extend and complement each other. Integrated systems have enabled more accurate modelling of domain knowledge, compensation for incomplete domain models and rule bases, compensation for small case bases, simplification of knowledge acquisition, improved solution quality, improved system efficiency, leveraging of past experiences, and compensation for shortcomings inherent in individual reasoning strategies. Thus integrations of $\mathrm{CBR}$ with other reasoning modalities continue to proliferate, providing both practical benefit and insight into multi-modal reasoning processes.

There are still a large number of important and challenging problems to be addressed in order to improve the quality and usefulness of expert systems for practical, real world problems. The research reported here has addressed the problem of how to achieve, and continually maintain, a higher level of competence and robustness in such systems than what they possess today. In "KNAPS-CR" systems, problem has been approached from two sides:

- Strengthening of the problem solving capability by combining several reasoning paradigms within a knowledge-rich environment, focusing on case-based reasoning as the major method.

- Enabling a continually improvement of an incomplete knowledge base by learning from each problem solving experience, using a knowledge-intensive, case-based learning method.

The resulting framework, architecture, system design, and representation platform - i.e. the "KNAPS-CR" approach - has been motivated and supported by relating it to strengths and weaknesses of other approaches

\section{REFERENCES}

[1] A. Aamoth and E. Plaza, "Case-Based Reasoning: Foundational Issues,Methodological Variations and System Approaches", Artificial Intelligence Communications, 7(1), 1994, pp. 39-59.

[2] Aamodt, A, 1994, Explanation-driven case-based reasoning. In Wess, S, Althoff, $\mathrm{K}$ and Richter, M (eds) Topics in Case-Based Reasoning. Berlin: Springer, pp. 274-288.

[3] Jackson P. (1999), Introduction to Expert Systems, Harlow, England: Addison Wesley Longman, Third Edition.

[4] Luger, George F., Artificial Intelligence: Structures and Strategies for Complex Problem Solving, Pearson Addison Wesley, Sixth Edition

[5] Russel S, and P. Norvig, (2002), Artificial Intelligence: A Modern Approach, Second Edition, Prentice- Hall, New Delhi.

[6] V.S.Janakiraman, K Sureshi, (2003), Artificial Intelligence and Expert Systems, Macmillan Series in Computer Science, New Delhi.

[7] Ramon López De Mántaras, David Mcsherry, Derek Bridge, David Leake, Barry Smyth, Susan Craw, Boi Faltings, Mary Lou Maher, Michael T. Cox, Kenneth Forbus, Mark Keane, Agnar Aamodt And Ian Watson "Retrieval, reuse, revision, and retention in casebased Reasoning",The Knowledge Engineering Review, Vol. 00:0, 1-2., Cambridge University Press, 2005

[8] I. Watson,"Case-based reasoning is a methodology not a technology, Knowledge-Based Systems", Vol. 12, 1999, pp 303-308

[9] Edwina L. Rissland and David B. Skalak, "Combining Case-Based and Rule-Based Reasoning: A Heuristic Approach”,pp534-530

[10] Stefanie Br"uninghaus and Kevin D. Ashley(2003), "Combining CaseBased and Model-Based Reasoning for Predicting the Outcome of Legal Case."

[11] Jim Prentzas and Ioannis Hatzilygeroudis ,"Categorizing Approaches Combining Rule-Based and Case-Based Reasoning"

[12] Cindy Marling, Edwina Rissland and Agnar Aamodt "Integrations with case-based reasoning" The Knowledge Engineering Review, Vol. 00:0, Cambridge University Press,2005 ,pp1-4.

[13] Jaap Hage ,"A Low Level Integration Of Rule-based Reasoning And Case-based Reasoning.”,pp30-39

[14] Z. Budimac, V. Kurbalija ,"CASE BASED REASONING - A SHORT OVERVIEW" ,Proceedings of the Second International Conference on Informatics and InformationTechnology,pp222-233 
[15] Bittencourt, I.I., M. Tadeu and E.B. Costa (2006) Combining AI techniques into a legal agent-based intelligent tutoring system, in Proceedings of the Eighteenth International Conference on Software Engineering and Knowledge Engineering (SEKE-2006), Skokie, IL: Knowledge Systems Institute, 35-40.

[16] Bogaerts, S., and Leake, D. 2005. A Framework for Rapid and Modular Case-Based Reasoning System Development. Technical Report TR 617, Computer Science Department, Indiana University, Bloomington, IN.

[17] Hanemann, L. (2006) A hybrid rule-based/case-based reasoning approach for service fault diagnosis, in Proceedings of the 2006
International Symposium on Frontiers in Networking with Applications, in conjunction with the Twentieth IEEE International Conference on Advanced Information Networking and Applications, Washington, DC: IEEE, 734-740.

[18] K. Ashwin Kumar, Yashwardhan Singh, Sudip Sanyal, (2009) Hybrid approach using case-based reasoning and rule-based reasoning for domain independent clinical decision support in ICU, Expert Systems with Applications 36, 65-71. 